\title{
The Effect of Quartz Window on Bistability of the Silicon Wafer in Lamp-Based Reactor
}

\author{
Valeriya P. Prigara, Aleksandr N. Kupriyanov, Vladimir V. Ovcharov* ${ }^{*}$ \\ Valiev Institute of Physics and Technology of Russian Academy of Sciences, Yaroslavl Branch, Yaroslavl, Russia \\ Email: *ovcharov.vlad@gmail.com
}

How to cite this paper: Prigara, V.P., Kupriyanov, A.N. and Ovcharov, V.V. (2020) The Effect of Quartz Window on Bistability of the Silicon Wafer in Lamp-Based Reactor. Journal of Materials Science and Chemical Engineering, 8, 54-65.

https://doi.org/10.4236/msce.2020.81006

Received: December 11, 2019

Accepted: January 13, 2020

Published: January 16, 2020

Copyright $\odot 2020$ by author(s) and Scientific Research Publishing Inc. This work is licensed under the Creative Commons Attribution International License (CC BY 4.0).

http://creativecommons.org/licenses/by/4.0/

\begin{abstract}
The effect of a quartz plate (window) on the silicon wafer temperature is studied in the conditions of the combined thermal transfer in a lamp-based chamber for the rapid thermal treatment (RTP) set up. The chamber for RTP is simulated by a radiative-closed thermal system including the influence of quartz window as a spectral filter of lamp emission and a source of emitted thermal radiation. Energy equations for thermal fluxes involved in the heat input and output from the working wafer and quartz window are solved in spectral approximation. The transfer characteristics that are defined by the temperature dependencies of the silicon wafer and the quartz window on the temperature of the heater are accounted. It is shown that temperature bistability in the silicon wafer initiates an induced bistability into the quartz window that does not reveal bistable behavior because of the linear temperature dependence of its total optical characteristics. A possibility for simulation of the quartz window by spectral restriction of the heater radiation is confirmed. The availability of the weak bistable effect in the mode of zero effective heat exchange coefficient of a non-radiative component of the thermal flux removed from the working wafer has been obtained.
\end{abstract}

\section{Keywords}

Lamp-Based Rector, Silicon Wafer, Quartz Window, Temperature and Optical Bistability, Induced Bistability Effect

\section{Introduction}

The processes of thermal treatment are an integral part of the micro- and nanoelectronics technology. A lamp-based chamber is one of the main units of the rapid treatment equipment that is widely used at present time for thermal processes including a post-implantation annealing [1] [2], diffuse doping [3] [4], 
crystallization of amorphous films [5] [6], contact annealing [7] [8], oxidation [9] [10] and etc. Thermal treatment processes differ in duration and radiation power from parts per million of second and peak power until $100 \mathrm{MW}$ on flesh-annealing [3] [4] to tens of seconds and radiation power $\sim 0.1 \mathrm{~kW}$ on low-temperature annealing [11]. On thermal treatment of a semiconductor wafer by high-powered radiation a temperature gradient along the depth of the wafer reaches very high values [2] and the wafer is exposed by powerful incoherent radiation fluxes transmitted through it. As a result, the wafer is far from the thermal equilibrium and together with non-linear optical properties of semiconductors these result in complicated thermal behavior of the wafer. The investigation of the silicon wafer behavior during its thermal process carried out in non-isothermal conditions for a lamp-based chamber makes it possible to predict theoretically and detect experimentally the hypothesis about the bistable behavior of the wafer [12]. The bistable behavior of the wafer proposes that in its thermal treatment process there is an interval of a controlling parameter (a heater temperature, as a rule) in which the wafer temperature takes different values according to whether the controlling parameter increases or decreases. A manifestation of the temperature and optical bistability phenomenon in a silicon wafer is caused by the non-linear temperature dependencies of its total emissivity and transmissivity. A stepwise increase of the optical characteristics is observed in the temperature interval from 600 to $800 \mathrm{~K}$ (see [12], for instance). As a consequence, the theoretical transfer characteristic of the working wafer (i.e., its temperature dependence on the temperature of the heater) has an $S$-like shape and is experimentally observed as a hysteresis loop [12]. Parameters of the hysteresis loop are determined by the optical properties of the thermal reactor elements, and the correspondence of theoretical and experimental transfer characteristics depends on the model of the reactor used in simulation. The thermal reactor in the system for the temperature-gradient heat treatment of semiconductor wafers is used for the experimental detection of the temperature bistability in the silicon wafer [12]. The detailed description of its construction has been presented in [12] [13]. The reactor includes a heating block and a working chamber. The working chamber is separated from the heating block by a quartz glass plate which is further referred to as "a quartz window". The chamber is filled by gas, as a rule, by argon or nitrogen. A water-cooled pedestal is located inside the working chamber. The silicon wafer is mounted on the special pins positioned on the water-cooled pedestal. The distance between the working wafer and the pedestal can be controlled by varying the height of the pins, then by controlling removal conductive flux from the wafer through a gas-filled gap to absorber. The model of the thermal chamber usually used in thermal simulation includes three plates: a heater plate, a working plate (wafer) and the absorber plate [14]. The quartz window influence, as a rule, is considered by restriction of the spectral interval for the heater emission in the spectral range from 0.4 to 4.0 $\mu \mathrm{m}$ [1] [15]. However, functions of the quartz window in the thermal system 
which models the lamp-based chamber extend further. The quartz plate is involved in the compound thermal exchange process. On the one hand, it is a semitransparent shield taking part in the exchange of thermal radiation between the heater and the working wafer, and on the other it is involved in convective energy transfer with the flow of air cooling the lamp block. Then, being part of the thermal system, the quartz influences the working wafer temperature. It absorbs, reflects, and reemits a radiative flux incident both to its face surface from the heater side and to its back from the working wafer side.

The object of the present investigation is analysis and comparison of different ways of simulating the lamp-based chamber and their impact on the shape of transfer characteristics that describe silicon wafer temperature dependence on the temperature of the heater modeling a lamp block of the thermal chamber. Particular attention has been given to the quartz window as a filter of radiation incident from the heater to the silicon wafer and as a source of emitted radiation.

\section{The Thermal Model of the Lamp-Based Chamber}

The thermal model of the non-isothermal lamp-based chamber includes four infinite-sized parallel-plane plates (Figure 1(a)). An upper plate (1) that is referred to as "the heater" models the block of tungsten-halogen lamps. The bottom plate (2) that is referred to as "the absorber" is meant for the simulation of walls and the bottom of the working chamber. Two semitransparent plates are located between the plates of the heater and the absorber. They are the protective shield, which is the quartz glass plate $(q)$, and the working wafer made from material possessing non-linear optical characteristics (silicon) $(f)$. The semitransparent plates have subscripted indexes $q$ and $f$, and their surfaces are marked by the second subscripted index. The surface turned to the heater and to the absorber are specified by the subscripts " 1 " and " 2 ", respectively.

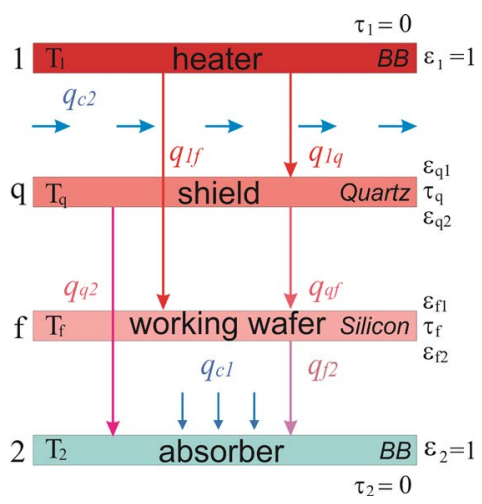

(a)

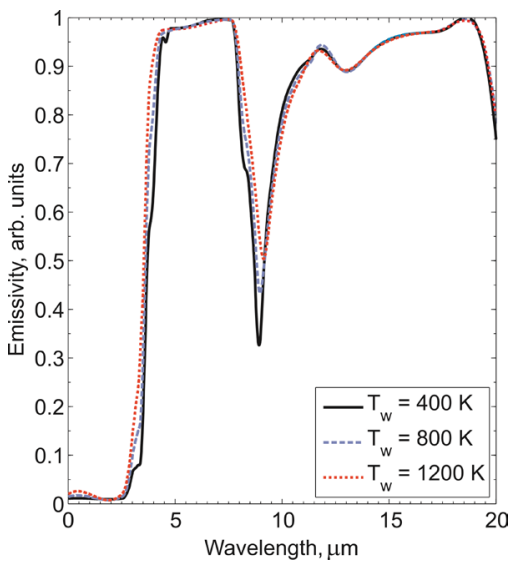

(b)

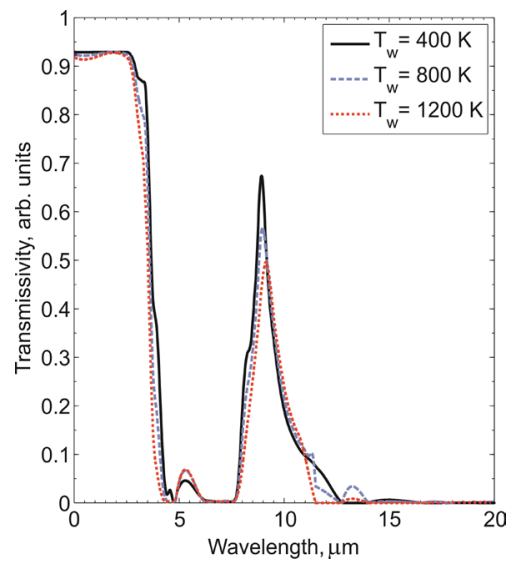

(c)

Figure 1. (a) Schematic representation of the thermal system model for lamp-heated working chamber with a quartz window cooled by a flow of compressed air; (b) (c) Spectral optical properties of the quartz window (QI type and $5 \mathrm{~mm}$ thick): (b) emissivity, (c) transmissivity. 


\section{Basic Equations}

The energy equations for thermal transfer between gray surfaces in such a thermal system are set up and solved in [16]. In [16] combined heat transfer is simulated in the radiative-closed thermal system. Besides radiative heat transfer in which all plates of the system take part, an extra heat removal is considered. It is the extra heat removal from the working wafer to the absorber plate by conductivity though the gas-filled gap between the wafer and the bottom plate of the absorber: $q_{c 1}=H_{\text {eff } 1}\left(T_{f}-T_{2}\right)$ and the forced convective heat removal from the quartz plate by flow of cooling air: $q_{c 2}=H_{e f f 2}\left(T_{q}-T_{g}\right)$. Here, $H_{e f f 1}, H_{e f f 2}$ are effective heat exchange coefficients [12]. $H_{\text {eff } 1}$ defines a non-radiative component of heat removal from the silicon wafer, and $H_{\text {eff } 2}$ is air-cooled degree of the quartz window. If the radiation fluxes between the heater and working wafer, the quartz plate and working wafer, the working wafer and absorber plate, the heater and quartz plate, and the quartz plate and absorber plate are denoted by $q_{1-f}, q_{q-f}, q_{f-2}, q_{1-q}$, and $q_{q-2}$, correspondingly, then the set of equations for the heat transfer in the thermal system modeling lamp-based chamber [16] can be rewritten as

$$
\left\{\begin{array}{l}
q_{1-f}+q_{q-f}=q_{f-2}+q_{c 1} \\
q_{1-q}=q_{q-f}+q_{q-2}+q_{c 2}
\end{array}\right.
$$

The gray surfaces approximation used in [16] is crude enough to describe thermal radiation heat transfer in the lamp-based chamber with regard to quartz glass window influence. The restriction of the upper bound of the heater radiation is exclusively spectral effect. So, we refine the gray approximation [16] by the spectral one:

$$
\left\{\begin{array}{l}
\int_{\lambda_{1}}^{\lambda_{2}} \mathrm{~d} q_{1-f}+\int_{\lambda_{1}}^{\lambda_{2}} \mathrm{~d} q_{q-f}=\int_{\lambda_{1}}^{\lambda_{2}} \mathrm{~d} q_{f-2}+q_{c 1} \\
\int_{\lambda_{1}}^{\lambda_{2}} \mathrm{~d} q_{1-q}=\int_{\lambda_{1}}^{\lambda_{2}} \mathrm{~d} q_{q-f}+\int_{\lambda_{1}}^{\lambda_{2}} \mathrm{~d} q_{q-2}+q_{c 2}
\end{array}\right.
$$

Here,

$$
\mathrm{d} q_{A-B}=\xi_{A-B}(\lambda, T) \cdot\left[e_{\lambda b}\left(\lambda, T_{A}\right)-e_{\lambda b}\left(\lambda, T_{B}\right)\right] \cdot \mathrm{d} \lambda
$$

where $\mathrm{d} q_{A-B}$ is radiation flux between $\mathrm{A}$ and $\mathrm{B}$ plates in the spectral range of $\mathrm{d} \lambda ; e_{\lambda b}(\lambda, T)$ is the hemispherical spectral emissive power for a surface of blackbody at temperature $T$ [17]; $\xi_{A-B}(\lambda, T)$ are spectral radiative coefficients for the heat transfer between A and B surfaces in terms of spectral optical data of the relevant plates: emissivity $\varepsilon(\lambda, T)$ and transmissivity $\tau(\lambda, T)$.

The following study is focused on the effect of the spectral characteristics of the silicon wafer and quartz glass plate on the heat transfer. Let us consider the problem in the first approximation when the heater and absorber plates possess optical properties of the blackbody $(\mathrm{ABB})\left(\varepsilon_{1}=\varepsilon_{2}=1 ; \tau_{1}=\tau_{2}=0\right)$. In this case, the relations between coefficients $\xi_{A-B}(\lambda, T)$ and coefficients $\varepsilon(\lambda, T)$ and $\tau(\lambda, T)$ are given by the same formulas as well as for the corresponding total characteristics in [16] $\left(\varepsilon_{1}=\varepsilon_{2}=1 ; \tau_{1}=\tau_{2}=0\right)$ : 


$$
\left\{\begin{array}{l}
\xi_{1-q}(T)=\frac{1}{d_{c}} \cdot\left\{\varepsilon_{q 1} \cdot\left(1-\left(1-\varepsilon_{q 2}-\tau_{q}\right) \cdot\left(1-\varepsilon_{f 1}-\tau_{f}\right)\right)+\varepsilon_{q 2} \tau_{q} \cdot\left(1-\varepsilon_{f 1}-\tau_{f}\right)\right\} \\
\xi_{q-f}(T)=\frac{1}{d_{c}} \cdot \varepsilon_{f 1} \cdot \varepsilon_{q 2} \\
\varepsilon_{q-2}(T)=\frac{1}{d_{c}} \cdot \varepsilon_{q 2} \cdot \tau_{f} \\
\xi_{1-f}(T)=\frac{1}{d_{c}} \cdot \varepsilon_{f 1} \cdot \tau_{q} \\
\xi_{f-2}(T)=\frac{1}{d_{c}} \cdot\left\{\varepsilon_{f 2} \cdot\left(1-\left(1-\varepsilon_{q 2}-\tau_{q}\right) \cdot\left(1-\varepsilon_{f 1}-\tau_{f}\right)\right)+\varepsilon_{f 1} \cdot \tau_{f}\right\} \\
d_{c}=1-\left(1-\varepsilon_{q 2}-\tau_{q}\right) \cdot\left(1-\varepsilon_{f 1}-\tau_{f}\right)
\end{array}\right.
$$

\section{Heat Transfer Simulation of the Thermal System Including the Quartz Window}

\subsection{The Transfer Characteristics of the Silicon Wafer}

The spectral optical data are used for quartz plate of QI type and $5 \mathrm{~mm}$ thick [18] [19] [20] in Figure 1(b) and Figure 1(c) and for $400 \mu \mathrm{m}$ thick working wafer from the lightly doped by boron silicon (100) whose optical characteristics are simulated by the use of the Rad-Pro program [21]. Let us restrict the spectral scale of wavelengths by the range from 0 to $20 \mu \mathrm{m}$ determined by the high intensity of the ABB emission. Figure 2(a) shows the three families of the transfer characteristics $T_{f}\left(T_{1}\right)$ at different values of $\mathrm{Si}$ wafer-cooling gas exchange coefficients $H_{\text {eff } 1}$ (each selected by the oval): 0,50 and $180 \mathrm{~W} /\left(\mathrm{m}^{2} \mathrm{~K}\right) . H_{e f f 1}$ is referred to further as a conductive heat exchange coefficient. To each value $H_{\text {eff } 1}$ correspond the following values of the quartz window gas exchange coefficient $H_{\text {eff } 2}: 0,80,200,700 \mathrm{~W} /\left(\mathrm{m}^{2} \mathrm{~K}\right)$, which form a family and determine the quartz

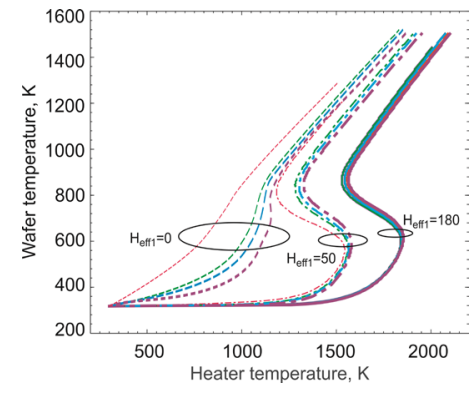

(a)

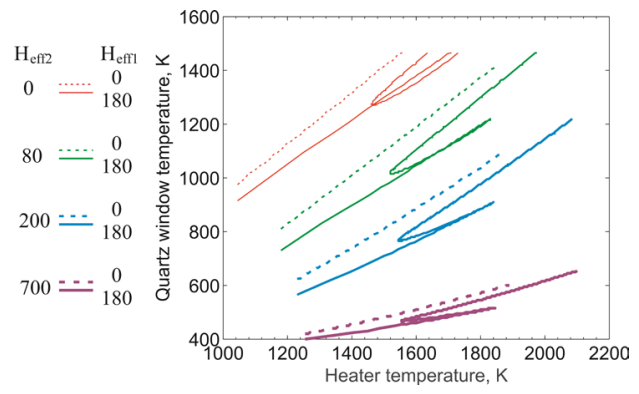

(b)

Figure 2. (a) Families of the transfer characteristics for the silicon wafer represented in Figure 1 (a) at the values of the conductive heat exchange coefficient $\left(H_{\text {eff } 1}\right): 0,50,180$ $\mathrm{W} /\left(\mathrm{m}^{2} \mathrm{~K}\right)$. In each family the convective heat exchange coefficient $H_{\text {eff } 2}$ takes the values $0,80,200,700 \mathrm{~W} /\left(\mathrm{m}^{2} \mathrm{~K}\right.$ ) (Cut off portions of the transfer curves at $H_{\text {eff } 2}=0$ and the absent curve at $H_{\text {eff } 1}=180 \mathrm{~W} /\left(\mathrm{m}^{2} \mathrm{~K}\right)$ and $H_{\text {eff } 2}=0$ are explained by the softening point of the quartz glass plate); (b) Families of the transfer characteristics for the quartz plate corresponding to curves from Figure 2(a) at $H_{\text {eff } 1}=0$ and $H_{\text {eff } 1}=180$ $\mathrm{W} /\left(\mathrm{m}^{2} \mathrm{~K}\right)$. 
window temperature on heating the working wafer. $H_{\text {eff } 2}$ is referred to further as a convective heat exchange coefficient. $H_{\text {eff } 1}=0$ corresponds to the merely radiation heat transfer between the working wafer and the absorber plate. At $H_{\text {eff } 1}=180 \mathrm{~W} /\left(\mathrm{m}^{2} \mathrm{~K}\right)$ the temperature bistability effect into the silicon wafer was experimentally observed in [12]. $H_{e f f 1}=50 \mathrm{~W} /\left(\mathrm{m}^{2} \mathrm{~K}\right)$ is an intermediate value between two formers. A family of the values of the convective gas exchange coefficient $H_{\text {eff } 2}$ allows us to estimate the quartz glass temperature effect on the transfer characteristics of the silicon wafer $T_{f}\left(T_{1}\right)$ on its heating. Figure 2 (a) shows that the temperature of the quartz plate exerts the most effect on the transfer characteristics of the silicon wafer in the mode when the extra mechanism of the heat removal from the silicon wafer is absent $\left(H_{e f f 1}=0\right)$. In the case when the convective gas exchange coefficient is as large as possible $H_{\text {eff } 2}=700$ $\mathrm{W} /\left(\mathrm{m}^{2} \mathrm{~K}\right)$ there is a sharp wafer temperature jump from low to high-temperature branches, which characterizes the behavior of the silicon wafer as the bistable one. The effect of the quartz window on the silicon wafer temperature loses force as conductive coefficient $H_{\text {eff } 1}$ increases: at $H_{\text {eff } 1}=180 \mathrm{~W} /\left(\mathrm{m}^{2} \mathrm{~K}\right)$ the varying convective coefficient results in negligible differences in the high-temperature parts of the transfer curves only.

\subsection{Transfer Characteristics of the Quartz Window}

Figure 2(b) shows the transfer characteristics of the quartz window in dependence on the heater temperature $T_{q}\left(T_{1}\right)$ for two values of the conductive heat exchange coefficient $H_{\text {eff } 1}: 0$ and $180 \mathrm{~W} /\left(\mathrm{m}^{2} \mathrm{~K}\right)$. As this takes place, the values of the convective heat exchange coefficient $H_{\text {eff } 2}$ are varied as in Figure 2(a). Figure 2(b) shows the high-temperature fragments of the transfer characteristics in the heater temperature range more than $1000 \mathrm{~K}$ because the portions of the transfer characteristics of the quartz plate increase near-linearly in the range from 300 to $1000 \mathrm{~K}$ and ones are not of special interest. Figure 2(b) shows that at value of the conductive heat exchange coefficient equal to null $\left(H_{\text {eff } 1}=0\right)$ the transfer curves of the quartz window are monotonically increasing curves (dotted lines) without any expressed singularities. Corresponding to them transfer curves of the silicon wafer $T_{f}\left(T_{1}\right)$ shown in Figure 2(a) are represented by the first family of the curves. From $H_{e f f 2}=80 \mathrm{~W} /\left(\mathrm{m}^{2} \mathrm{~K}\right)$ these curves have the portions when the temperature of the silicon wafer $T_{f}$ increases reasonably sharply as the heater temperature increases. Figure 2(a) shows that at $H_{\text {eff } 1}=0$ there is one curve that has hysteresis loop with arbitrarily small width. It corresponds to $H_{\text {eff } 2}=700 \mathrm{~W} /\left(\mathrm{m}^{2} \mathrm{~K}\right)$. A completely different situation is observed for transfer curves with conductive efficient heat exchange coefficient equal to $H_{\text {eff } 1}=180 \mathrm{~W} /\left(\mathrm{m}^{2} \mathrm{~K}\right)$. Each of them is defined as the well-marked hysteresis loop of $S$-like shape. Figure 2(b) shows that to transfer curves for silicon wafer $T_{f}\left(T_{1}\right)$ correspond the transfer curves for the quartz plate $T_{q}\left(T_{1}\right)$ the shape of which is smoothly changed from loop-like at $H_{\text {eff } 2}=0$ to $S$-like shape at $H_{\text {eff } 2}=700 \mathrm{~W} /\left(\mathrm{m}^{2} \mathrm{~K}\right)$. The width and the location of these loops for the quartz plate precisely correspond to the width and localization of the relevant loops for 
the silicon wafer Figure 2(a). As the optical properties of the quartz glass plate do not show non-linear properties [22], the hysteresis loops of $T_{q}\left(T_{1}\right)$ have induced nature.

\subsection{Discussion}

Analysis of Figure 2(a) and Figure 2(b) enables to explain behavior regularities of the transfer curves $T_{f}\left(T_{1}\right)$ in the limits of each curve family at fixed conductive heat exchange coefficient $H_{\text {eff } 1}$ and at varying values of the convective heat exchange coefficient $H_{\text {eff } 2}$ (Figure 2(a)). Because the quartz plate transmits in the spectral range from 0 to $4 \mu \mathrm{m}$ while it is practically opaque in the remainder part of the interesting for us spectrum range from 0 to $20 \mu \mathrm{m}$ (for exception of the peak near $9 \mu \mathrm{m}$ ), it behaves as an opaque shield with the spectral window in the range 0 to $4 \mu \mathrm{m}$. The shield can be considered as a composed heater with relation to working wafer: it emits as an $\mathrm{ABB}$ heater at the heater temperature $T_{1}$ in the spectral range from 0 to $4 \mu \mathrm{m}$, and one does as the source of emitted radiation at quartz plate temperature $T_{q}$ in the spectral range from 4 to $20 \mu \mathrm{m}$. Figure 2(b) shows that at small values of convective heat exchange coefficient $H_{\text {eff } 2}$ the temperature of the quartz plate run to $800-900 \mathrm{~K}$ for the family of the transfer curves $T_{q}\left(T_{1}\right)$ corresponding to zero conductive heat exchange coefficient $\left(H_{\text {eff } 1}=0\right)$ and to heater temperature increasing from 300 to $1000 \mathrm{~K}$. Thus, the emission of the quartz plate differs weakly from the heater emission. Therefore, the behavior of the relevant transfer characteristics $T_{f}\left(T_{1}\right)$ is only weakly distinguished from the behavior of the same characteristics simulated using the model in which the quartz glass plate is absent. As the values of convective heat exchange coefficient $\left(H_{\text {eff } 2}\right)$ increases, the temperature of the silicon wafer is reduced (Figure 2(b)), and with it, the efficiency of the quartz plate as the heater in the range from 4 to $20 \mu \mathrm{m}$ is also reduced. This leads to progressive formation of the obviously defined low-temperature branch on the transfer characteristics $T_{f}\left(T_{1}\right)$ and increasingly slanting its tracing. As a result the low-temperature branch of the transfer curve at $H_{\text {eff } 2}=700 \mathrm{~W} /\left(\mathrm{m}^{2} \mathrm{~K}\right)$ is delayed followed by the temperature jump-up in the silicon wafer as large as $150 \mathrm{~K}$ at $T_{1} \sim 1200 \mathrm{~K}$. The low-temperature branches of the transfer curves $T_{f}\left(T_{1}\right)$ at $H_{\text {eff } 1}=50$ and $180 \mathrm{~W} /\left(\mathrm{m}^{2} \mathrm{~K}\right)$ are practically merged at all values of $H_{\text {eff } 2}$. This is due to the reasonably large efficiency of the non-radiative component from the working wafer to absorber. It levels the difference in heat input to wafer at varying $H_{\text {eff } 2}$. The high-temperature branches of the transfer curves $T_{f}\left(T_{1}\right)$ at all values of the conductive heat exchange coefficient $H_{\text {eff } 1}$ tend to have the same regularities: the larger $H_{\text {eff } 2}$ the further shifted the relevant branch to the right along the scale of the heater temperature.

\section{Comparison between the Silicon Wafer Transfer Curves Simulated by Different Models of the Quartz Window}

To understand the quartz window influence on the transfer curves of the ther- 
mal system we compare the transfer curves that are simulated by the model composed of three plates (Figure 3(a)) [12] (the heater, the working wafer and the absorber) and by the model which additionally include the quartz window (Figure 1(a)). Figure 3(b) shows the transfer characteristics calculated by use of the model including three plates [12] (solid lines) where the heater emits in the range of wavelengths from 0 to $20 \mu \mathrm{m}$; by the corrected model including three plates [12] (dash-and-dotted curves) where the heater emits in the spectral range from 0 to $4 \mu \mathrm{m}$ [14] [15], and by the model with the quartz window (dashed curves) where the heater emits in the range 0 to $20 \mu \mathrm{m}$. Contrary to [12], where the spectral data are calculated by the approximating relations, here, the spectral characteristics are obtained by the use of the Rad-Pro program [21]. The quantity of the convective heat exchange coefficient $H_{\text {eff } 2}=700 \mathrm{~W} /\left(\mathrm{m}^{2} \mathrm{~K}\right)$ that defines heat removal from the quartz window by the flow of compressed air is chosen large enough so as to correspond to the experimental conditions excluding the overheating the quartz glass plate. As discussed above, the quartz window can be considered as the composed heater, which transmits radiation of the $\mathrm{ABB}$ heater in the range from 0 to $4 \mu \mathrm{m}$ and gives emitted radiation from 0 to 20 $\mu \mathrm{m}$. As the efficiency of heat removal from the quartz plate by compressed air flow increases, i.e. as $H_{e f f 2}$ increases, the temperature of the quartz window and its emitted radiation decreases. The model of the chamber composed of the three plates with different spectral ranges of the heater radiation can be considered as two limiting cases of the chamber model including the quartz window. In one limiting case, when emitted radiation of the quartz plate is equal to zero,

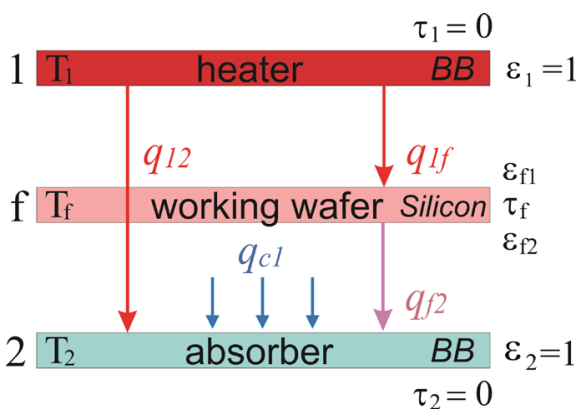

(a)

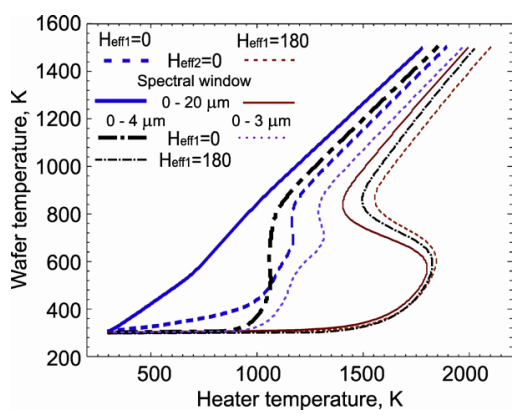

(b)

Figure 3. (a) Schematic representation of the thermal system model for the lamp-based working chamber composed of the three plates [12] (without quartz plate): the ABB heater plate (spectral interval of the heater emission can be varied), the working silicon wafer (a thin plate) and ABB absorber plate; (b) The silicon wafer transfer curves simulated for thermal system model with the quartz window at $H_{\text {eff } 1}=0, H_{\text {eff } 2}=700$ (thick dotted line), at $H_{e f f 1}=180 \mathrm{~W} /\left(\mathrm{m}^{2} \mathrm{~K}\right)$ and $H_{e f f 2}=700$ (thin dotted line), the silicon wafer transfer curves for thermal system model without the quartz window in the spectral range from 0 to $20 \mu \mathrm{m}$ at $H_{e f f 1}=0$ (thick solid line) and at $H_{e f f 1}=180 \mathrm{~W} /\left(\mathrm{m}^{2} \mathrm{~K}\right.$ ) (thin solid line); ones for the restriction of the heater emission spectral interval in the range 0 to 4 $\mu \mathrm{m}$ at $H_{e f f 1}=0$ (thick dot-and-dash line); at $H_{e f f 1}=180 \mathrm{~W} /\left(\mathrm{m}^{2} \mathrm{~K}\right)$ (thin dotted line) and for the restriction of the heater emission spectral interval in the range 0 to $3 \mu \mathrm{m}$ at $H_{e f f 1}=0 \quad$ (spotted line). 
we obtain the chamber model composed of three plates with the heater that radiates in the range 0 to $4 \mu \mathrm{m}$. In the other limiting case, the emitted radiation of the quartz plate can be considered as the $\mathrm{ABB}$ heater radiation in the range 4 to $20 \mu \mathrm{m}$. Such an approach provides insight into reciprocal location and singularity of the transfer curves $T_{f}\left(T_{1}\right)$ represented in Figure 3(b). As is indicated in the figure, the low-temperature branches of the transfer curves of the silicon wafer at low values of $H_{\text {eff } 1}$ are especially strongly dependent on the restriction of the spectral interval of the emission of $\mathrm{ABB}$ heater from 0 to $4 \mu \mathrm{m}$ in the model composed of three plates and via the quartz window in the model composed of four plates. The high-temperature branches of the transfer characteristics that account for $H_{\text {eff } 2}=0$ и $H_{\text {eff } 2}=700 \mathrm{~W} /\left(\mathrm{m}^{2} \mathrm{~K}\right)$ are shifted in approximately the same distances with respect to each other, whereas their low-temperature branches vary widely. The sharp stepwise jump of the silicon wafer at the heater temperature $\sim 1200 \mathrm{~K}$ brings the behavior of the silicon wafer at $H_{\text {eff } 1}=0$ и $H_{\text {eff } 2}=700 \mathrm{~W} /\left(\mathrm{m}^{2} \mathrm{~K}\right)$ closer to bistable behavior, whereas the transfer curves $T_{f}\left(T_{1}\right)$ at $H_{\text {eff } 1}=H_{\text {eff } 2}=0$ rises from linear law practically as the heater temperature $T_{1}$ increases. The non-linear increase of $T_{f}\left(T_{1}\right)$ is vividly revealed in the case when the lamp chamber is modeled by three plates and emission of its heater is restricted by the range of $0-4 \mu \mathrm{m}$. The low-temperature branch of this silicon wafer transfer curve is terminated at the heater temperature $\sim 1070 \mathrm{~K}$ by the stepwise change of the wafer temperature near on $300 \mathrm{~K}$. The jump is given rise by low-temperature branch tightening caused by underheated of the wafer because of the absolute exclusion of the heater emission in the range of $4-20$ $\mu \mathrm{m}$. It should be mentioned that within the limits of interpretation of quartz glass as a composed heater the discrepancy occurs. The relevant transfer characteristic $T_{f}\left(T_{1}\right)$ described by the model of three plates with the restriction of the spectral interval of the heater emission is to be positioned to the right of the transfer characteristics obtained by the use of the model of four plates at the different values of $H_{\text {eff } 2}$. Such a discrepancy between the locations of these curves is correlated to the wider spectral interval by which the bandwidth for heater emission transmitted though the quartz window is modeled. Figure 1(c) shows the family of curves in the spectral range from 0 to $20 \mu \mathrm{m}$. They are the spectral transmissivity of the quartz plate of QI type and $5 \mathrm{~mm}$ thick at the indicated temperatures. Referring to the figure, the quartz plate bandwidth lies in the range 0.4 to $3 \mu \mathrm{m}$ and at the level of 0.92 . In the spectral range from 3 to $4 \mu \mathrm{m}$ the transmission of the quartz window falls to zero. For checking this hypothesis the transfer characteristic $T_{f}\left(T_{1}\right)$ is plotted using the chamber model of three plates and heater emission in the range from 0 to $3 \mu \mathrm{m}$ at $H_{\text {eff } 1}=0$ (Figure $3(\mathrm{~b})$ ). As expected, it is disposed to the right of the relevant transfer characteristics plotted by model including four plates. A similar result should be expected for transfer characteristics at $H_{\text {eff } 1}=180 \mathrm{~W} /\left(\mathrm{m}^{2} \mathrm{~K}\right)$.

A comparison between the transfer characteristics shown in Figure 3(b) at $H_{\text {eff } 1}=0$ and the experimental transfer curves obtained in [12] at low values $H_{e f f 1}$ shows that the sharp jump of the silicon wafer temperature in the dotted, 
dash-and-dotted and spotted curves is caused by the optical properties of the blackbody heater and filtering its radiation in the range of wavelengths over 4 $\mu \mathrm{m}$. The sharp increasing radiation flux incident on the silicon wafer from the side of the blackbody heater on being heated to the temperatures above $1000 \mathrm{~K}$ is related to advancing maximum of the energy that is emitted by the blackbody heater in the 'allowed' range of wavelength from 0 to $4 \mu \mathrm{m}\left(\lambda_{\max }=3 \mu \mathrm{m}\right.$ at $T_{1}=1000 \mathrm{~K}$ ). In this case, the efficiency of the radiative heat removal from the silicon wafer in the low-temperature state is insufficient that the wafer is maintained in the stationary state. Besides, the carried out calculations show that filtering radiation of the heaters that possess the optical properties which are close to the optical properties of the blackbody (silicon carbide, for example) can initiate complicated behavior in semiconductor structures when they interact with such a radiation under ordinary conditions without extra forced heat removal. As a result, the wafer is step-wise switched to the high-temperature state.

\section{Conclusion}

Thus, the numerical solutions of equations were received for the description of the combined heat transfer in the radiative-closed thermal system composed of four plates, two interior of them are semitransparent for thermal radiation and outer ones possess the optical properties of blackbody. These equations are spectral modification of the equations that are received in [16] for the total approximation of gray bodies. The transfer characteristics of the silicon wafer and quartz window are simulated in the thermal system modeling the lamp-based chamber for the different values of the heat exchange coefficients defining non-radiative components of thermal fluxes removed from the silicon wafer and quartz plate. It is shown that the quartz window that separates the work zone of the chamber from the heating block can be considered as the composed heater, whose emission is determined by $\mathrm{ABB}$ heater in the spectral range from 0 to 4 $\mu \mathrm{m}$ and by emitted radiation of the quartz plate in the spectral range from 4 to $20 \mu \mathrm{m}$. The efficiency of the emitted radiation of the quartz plate decreases as the cooling air flow (that is characterized by the convective heat exchange coefficient $H_{\text {eff } 2}$ ) and, therefore, radiation heat flux removed from the quartz plate increase. The shape of the silicon wafer transfer curves $T_{f}\left(T_{1}\right)$ is strongly dependent on the value of $H_{\text {eff } 2}$ when the nonradiative component of the heat flux that is transferred from the silicon wafer to the absorber is absent. At the large values of convective heat exchange coefficient $H_{\text {eff } 2}$ the behavior of the silicon wafer is near to a bistable one even at $H_{\text {eff } 1}=0$. The transfer curves of the quarts plate $T_{q}\left(T_{1}\right)$ depend on the configuration of the transfer curves of the silicon wafer. In the case when the hysteresis loop appears on the transfer characteristics of the silicon wafer $T_{f}\left(T_{1}\right)$ the hysteresis loop of the same width and at the same controlling parameters arises on the transfer curves of the quartz window $T_{q}\left(T_{1}\right)$. The width and the locations of the loops are exactly in accordance with the same parameters for the silicon wafer transfer characteristics. 
This result allows to interpret of such a phenomenon as induced bistability effect in the quartz plate material which does not have the relevant non-linear optical properties for the revealing the bistability effect. The obtained results can be a basis for simulating the quartz plate effect on the silicon wafer temperature in the lamp-based chambers of RTA set-up in conditions of real spectral characteristics of thermal radiation for the heater and absorber. Besides, the carried out calculations show that filtering the radiation of the heaters that possess optical properties which are close to the ones of the blackbody (a heater made from silicon carbide, for instance) can initiate a complicated behavior in semiconductor structures at their interaction with such a radiation under ordinary conditions without extra forced cooling.

\section{Conflicts of Interest}

The authors declare no conflicts of interest regarding the publication of this paper.

\section{References}

[1] Timans, P., Xing, G., Hamm, S., McCoy, S., Cibere, J., Stuart, G. and Camm, D. (2012) Flat-Top Flash Annealing ${ }^{\text {Tm }}$ for Advanced CMOS Processing. In: 12th International Workshop on Junction Technology, IEEE, Piscataway, 63-68.

https://doi.org/10.1109/IWJT.2012.6212811

[2] McMahon, R.A., Smith, M.P., Seffen, K.A., Voelskow, M., Anwand, W. and Skorupa, W. (2007) Flash-Lamp Annealing of Semiconductor Materials-Applications and Process Models. Vacuum, 81, 1301-1305. https://doi.org/10.1016/j.vacuum.2007.01.033

[3] Prucnal, S., Rebohle, L. and Skorupa, W. (2017) Doping by Flash Lamp Annealing. Materials Science in Semiconductor Processing, 62, 115-127. https://doi.org/10.1016/j.mssp.2016.10.040

[4] Ho, J.C., Yerushalmi, R., Jacobson, Z.A., Fan, Z., Alley, R.L. and Javey, A. (2008) Controlled Nanoscale Doping of Semiconductors via Molecular Monolayers. Nature Materials, 7, 62-67. https://doi.org/10.1038/nmat2058

[5] Tüzün, Ö., Slaoui, A., Roques, S., Focsa, A., Jomard, F. and Ballutaud, D. (2009) Solid Phase Epitaxy on N-Type Polysilicon Films Formed by Aluminium Induced Crystallization of Amorphous Silicon. Thin Solid Films, 517, 6358-6363. https://doi.org/10.1016/j.tsf.2009.02.091

[6] Jennings, D., Mayur, A., Parihar, V., Haifan Liang, Mcintosh, R., Adams, B., Thakur, R., et al. (2004) Dynamic Surface Anneal: Activation without Diffusion. In: 12 th IEEE International Conference on Advanced Thermal Processing of Semiconductors, IEEE, Piscataway, 47-51.

[7] Chi, D.Z., Lee, R.T.P. and Wong, A.S.W. (2007) Addressing Materials and Integration Issues for NiSi Silicide Contact Metallization in Nano-Scale CMOS Devices. Thin Solid Films, 515, 8102-8108. https://doi.org/10.1016/j.tsf.2007.02.020

[8] Yu, H., Schaekers, M., Rosseel, E., Peter, A., Lee, J.-G., Song, W.-B., De Meyer, K., et al. (2015) $1.5 \times 10^{-9} \Omega \mathrm{cm}^{2}$ Contact Resistivity on Highly Doped Si:P Using Ge Pre-Amorphization and Ti Silicidation. In: IEEE International Electron Devices Meeting, IEEE, Piscataway, 21-27. https://doi.org/10.1109/IEDM.2015.7409753

[9] Constant, A., Camara, N., Godignon, P., Berthou, M., Camassel, J. and Decams, J. 
M. (2010) Rapid and Efficient Oxidation Process of SiC by in Situ Multiple RTP Steps. Materials Science Forum, 645, 817-820. https://doi.org/10.4028/www.scientific.net/MSF.645-648.817

[10] Kosugi, R., Fukuda, K. and Arai, K. (2005) High Temperature Rapid Thermal Oxidation and Nitridation of $4 \mathrm{H}-\mathrm{SiC}$ in Diluted $\mathrm{N}_{2} \mathrm{O}$ and $\mathrm{NO}$ Ambient. Materials Science Forum, 483, 669-672.

https://doi.org/10.4028/www.scientific.net/MSF.483-485.669

[11] Alptekin, E., Ozturk, M.C. and Misra, V. (2009) Tuning of the Platinum Silicide Schottky Barrier Height on n-Type Silicon by Sulfur Segregation. IEEE Electron Device Letters, 30, 331-333. https://doi.org/10.1109/LED.2009.2014182

[12] Rudakov, V., Ovcharov, V., Kurenya, A. and Prigara, V. (2012) Bistable Behavior of Silicon Wafer in Rapid Thermal Processing Setup. Microelectronic Engineering, 93, 67-73. https://doi.org/10.1016/j.mee.2011.11.018

[13] Mochalov, B.V. and Rudakov, V.I. (1996) A Setup for the Temperature-Gradient Heat Treatment of Semiconductor Wafers. Instruments and Experimental Techniques, 39, 302-304.

[14] Fiory, A.T. (2005) Rapid Thermal Processing for Silicon Nanoelectronics Applications. JOM, 57, 21-26. https://doi.org/10.1007/s11837-005-0131-0

[15] Merchant, T.P., Cole, J.V., Knutson, K.L., Hebb, J.P. and Jensen, K.F. (1996) A Systematic Approach to Simulating Rapid Thermal Processing Systems. Journal of the Electrochemical Society, 143, 2035-2043. https://doi.org/10.1149/1.1836945

[16] Ovcharov, V. and Prigara, V. (2019) Induced Bistability into Quartz Glass by Silicon Wafer Heat Treatment in Lamp-Based Reactor. Proceedings of SPIE, Vol. 11022, 110221U-1. https://doi.org/10.1117/12.2521891

[17] Siegel, R. and Howell, J.R. (1992) Radiation Heat Transfer. Hemisphere Publ. Corp., Washington DC.

[18] Petrov, V.A., Stepanov, S.V. and Muchamedyarov, K.S. (1983) Tablitsi Standartnih Spravochnih Dannih [Standard Reference Tables] GSSSD-40-82. Gosstandart, Moscow.

[19] Petrov, V.A., Stepanov, S.V. and Muchamedyarov, K.S. (1982) Tablitsi Standartnih Spravochnih Dannih [Standard Reference Tables] GSSSD-26-81. Gosstandart, Moscow,

[20] Petrov, V.A. and Stepanov, S.V. (1975) Radiation Characteristics of Quartz Glasses Spectral Radiating Power. High Temperature, 13, 308-316.

[21] Lee B.J. and Zhang, Z.M. (2005) Rad-Pro: Effective Software for Modeling Radiative Properties in Rapid Thermal Processing. In: 13th International Conference on Advanced Thermal Processing of Semiconductors, IEEE, Piscataway, 275-281.

[22] Petrov, V.A. and Stepanov, S.V. (1975) Radiation Characteristics of Fused Quartz-Integrated Emissivity. High Temperature, 13, 1092-1099. 